\title{
Análisis funcional de Consolación del Sur y Sandino como centros intermedios del sistema de asentamientos humanos de Pinar del Río, Cuba
}

\section{Functional analysis of Consolacion del Sur and Sandino as intermediate centers of the human settlement system of Pinar del Rio, Cuba}

\author{
Juan P. Cabrera Díaz* \\ Isabel M. Valdivia Fernández* \\ Dunia Cabrera Díaz**
}

Fecha de recibido: 11 de diciembre de 2018

Fecha de aceptado: 14 de agosto de 2019

\section{Resumen}

Existe una gran variedad de factores que afectan la configuración de los territorios. El sistema de asentamientos humanos, la estructura económica, su historia, conectividad, infraestructura y servicios son algunos de los más importantes. En este trabajo, se realiza un examen de varios de estos factores en la provincia, para determinar la funcionalidad de las ciudades como centros intermedios del sistema de asentamientos humanos.

Con base en los resultados de esta investigación, se pueden hacer cambios en los esquemas y planes de pedidos de estos asentamientos, los subsistemas territoriales que conforman la provincia. Finalmente, se propone un grupo de acciones o políticas que se llevarán a cabo en cada uno de estos asentamientos para mejorar su funcionalidad y tratar de eliminar la dependencia que tienen la mayoría de los pueblos de la capital provincial, un aspecto que establece un

\footnotetext{
Facultad de Geografía, Universidad de La Habana, Cuba, correos electrónicos: juan.pablo@geo.uh.cu; ival@geo.uh.cu

* Universidad de Pinar del Río, Cuba, correo electrónico: dunia@upr.edu.cu
} 
carácter monocéntrico en términos de cuestiones socioeconómicas y administrativas en ese territorio.

Palabras claves: territorio, sistema de asentamientos humanos, planificación.

\section{Abstract}

There is a great variety of factors that affect the configuration of the territories. The human settlements system, the economic structure, its history, connectivity, infrastructure and services are some of the most important. In this work, an examination of several of these factors is made in the province in order to determine the functionality of these cities as intermediate centers of the human settlements system.

Based on the results of this research, changes can be made to the schemes and ordering plans of these settlements, the territorial subsystems that make up and the province. Finally, a group of actions or policies to be carried out in each of these settlements are proposed to improve their functionality and try to eliminate the dependency that most of the towns in the provincial capital have, an aspect that establishes a monocentric character in terms of socioeconomic and administrative issues in that territory.

Key words: territory, human settlements system, planification.

\section{Introducción}

La provincia de Pinar del Río, ubicada al extremo occidental de Cuba, posee una red de servicios en su sistema territorial que tributa al sistema de asentamientos humanos. Es por ello que ambos sistemas están estrechamente relacionados, aunque también poseen sus características distintivas.

La misma posee una red de servicios poco diversificada, donde solo la capital provincial presenta un amplio desarrollo en este sentido, siendo capaz de abastecer a los demás territorios de la provincia. Este aspecto supone una problemática por la cantidad de personas que se desplazan diariamente hacia ese centro polarizador, pues su región de origen no tiene las condiciones para ofrecerles el servicio que estos están buscando aunque, en muchos casos, son servicios de necesidad vital. Es por ello que podemos afirmar el carácter monocéntrico de los servicios en la provincia y el poder polarizador de la ciudad cabecera.

Pinar del Río, además de tener un amplio territorio, no presenta un elevado desarrollo del transporte, ni tiene facilidades de accesibilidad por las causas anteriores. Estos aspectos imposibilitan la funcionalidad del sistema de asentamientos y resaltan la importancia de la necesidad del correcto funcionamiento de sus centros intermedios. 
Pinar del Río cuenta, según los análisis y programas desarrollados por la Dirección Provincial de Planificación Física (DPPF), con dos asentamientos declarados centros intermedios, que son Sandino en la región Occidental y Consolación del Sur en la región Oriental.

Este tema tiene gran importancia pues se inserta en las líneas de ordenamiento territorial de la provincia y en el programa de desarrollo socioeconómico territorial. Es un estudio novedoso pues vincula dos sistemas muy importantes que interactúan en el territorio, el sistema de asentamientos humanos y el sistema territorial de los servicios. La articulación entre ambos es vital para fomentar el desarrollo de los territorios, además de mejorar los niveles de acceso de las personas a los servicios y su bienestar.

\section{Objetivo}

Analizar el funcionamiento de las ciudades de Consolación del Sur y Sandino como centros intermedios del sistema de asentamientos humanos en Pinar del Río.

En el caso cubano se denomina centros intermedios (Bermúdez, 2015), a los asentamientos humanos que asumen este tipo de relación con un territorio determinado (subsistema), debido a que incluye asentamientos que no son considerados ciudades, por su talla poblacional, sin embargo tienen y deben tener una función territorial de intermediación con otros asentamientos (ciudades, pueblos, poblados y caseríos) y con la población dispersa. En la actualidad se potencia el desarrollo de 32 ciudades que se insertan en esta clasificación.

Para alcanzar los principales resultados en esta investigación fue necesario tener en cuenta la teoría clásica de Christaller sobre los lugares centrales. Este autor proponía tres elementos fundamentales para ser aplicados los centros intermedios de Pinar del Río y comprobar su correcto funcionamiento:

- Centralidad de los mercados

- El sistema de transporte

- El poder administrativo

Según el Programa Provincial de Ordenamiento Territorial (PPOT) realizado por la DPPF en 2014, encontramos cuatro regiones funcionales, partiendo de la conectividad entre los asentamientos y de la funcionalidad de los mismos como se muestra en la Figura 1.

Se procedió entonces al análisis de la funcionalidad de los centros intermedios del sistema de asentamientos teniendo en cuenta los tres elementos propuestos por Christaller. 


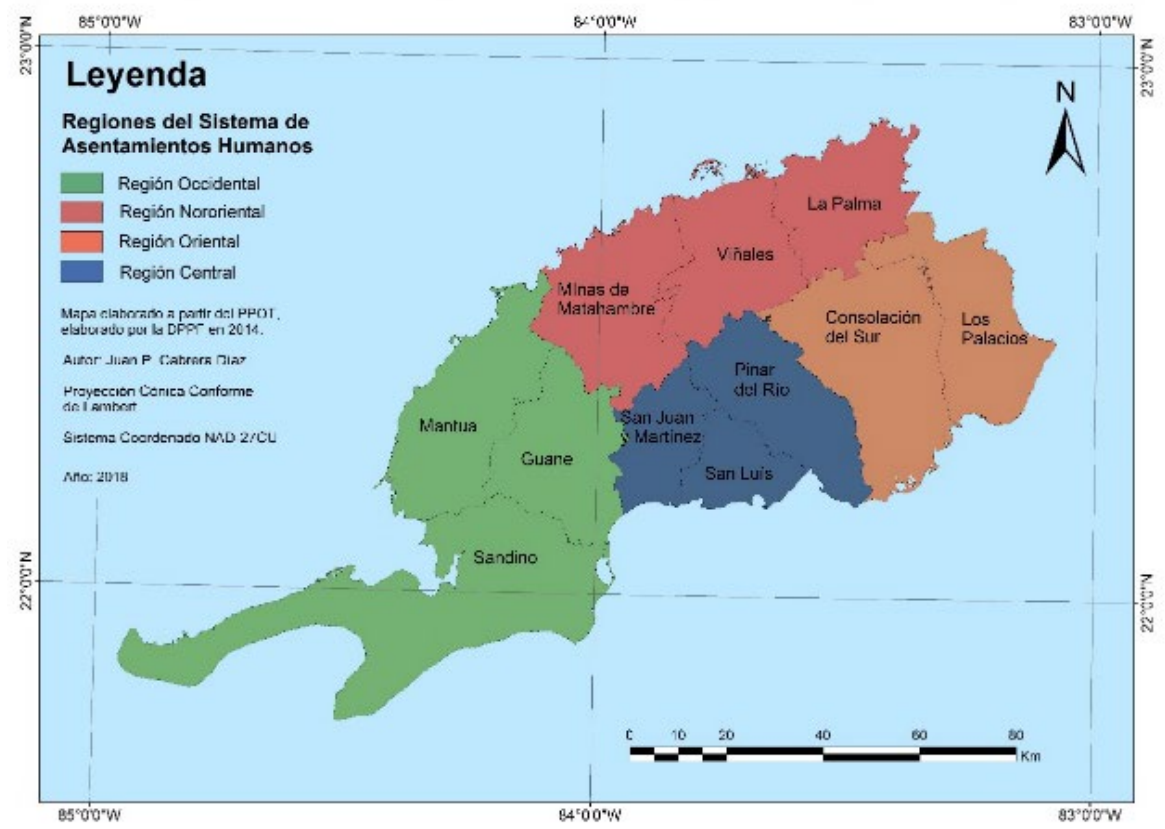

Figura 1. Regionalización funcional del Sistema de Asentamientos de la provincia Pinar del Río.

Fuente: elaborado por el autor.

\section{Análisis de centralidad, conectividad y jerarquía de los asentamientos sandino y consolación del sur}

Para establecer la centralidad, se utilizó el Índice de Davies. A partir de los datos de centralidad calculados mediante el Índice de Davies para cada uno de los municipios de cada región, se determinó el alcance territorial de la centralidad de cada cual. Para asignar el peso se calcularon los radios de los círculos correspondientes al área de cada territorio y finalmente se determinó la relación entre las magnitudes de estos radios, relacionando el valor del índice con el área de la provincia objeto de estudio (Figura 2).

Este mapa resalta el carácter monocéntrico de la provincia y destaca la situación negativa que existe en cuanto a los servicios en las regiones Occidental y Nororiental.

El resultado final del análisis de la centralidad determina que Consolación del Sur presenta las potencialidades para funcionar como centro intermedio. En el caso de la región Occidental no presenta, según los niveles de centralidad calculado, una ciudad que tenga las condiciones para generar una atracción para satisfacer la demanda de los servicios necesarios para la población. La 


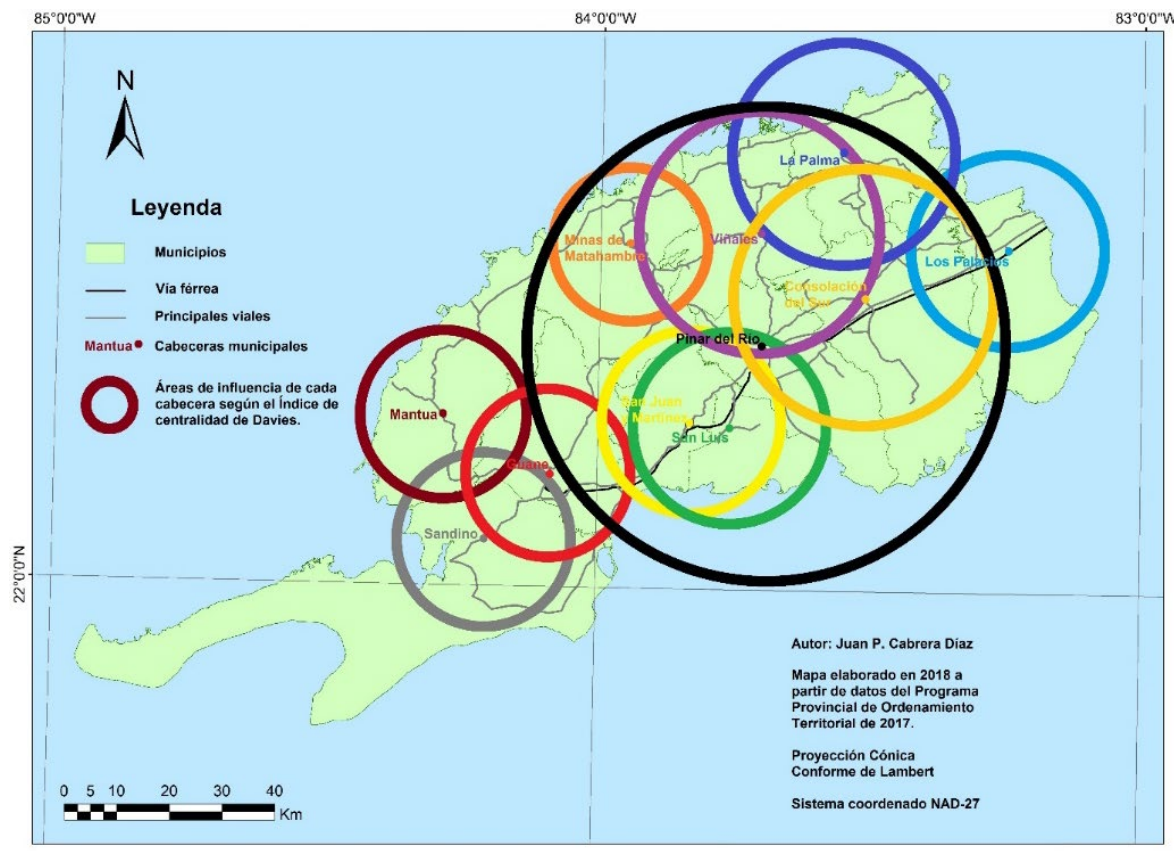

Figura 2. Mapa de centralidad de la provincia Pinar del Río.

Fuente: elaborado por el autor.

conectividad se analizó según la Teoría de los Grafos. No se puede pasar por alto el fuerte nivel de deterioro que presentan las mismas, en especial en los casos de menor orden jerárquico, aunque presenten alto grado de explotación. Los resultados sobre los diferentes aspectos que determinan la conectividad entre los asentamientos de la provincia Pinar del Río se resume en la Figura 3.

La provincia de Pinar del Río cuenta con 452 asentamientos humanos concentrados, de los cuales 43 son urbanos y 409 rurales. Estas clasificaciones responden a los requisitos establecidos en la división político-administrativa del año 2011, la cual determinó algunos cambios en la estructura territorial de la provincia. El mismo DPPF (2014), establece la jerarquía de los principales asentamientos humanos a partir del análisis del nivel y cobertura de los servicios en esos territorios. El resultado se presenta en la Figura 4.

Al analizar cómo se encuentra estructurado el sistema de asentamientos humanos de la provincia, el resultado se observa en la Figura 5.

Como se puede apreciar el estimado muestra cómo debe presentarse las isolíneas de distribución de la población teniendo en cuenta los valores establecidos de cantidad de habitantes en cada uno de los principales asentamientos de la provincia. 


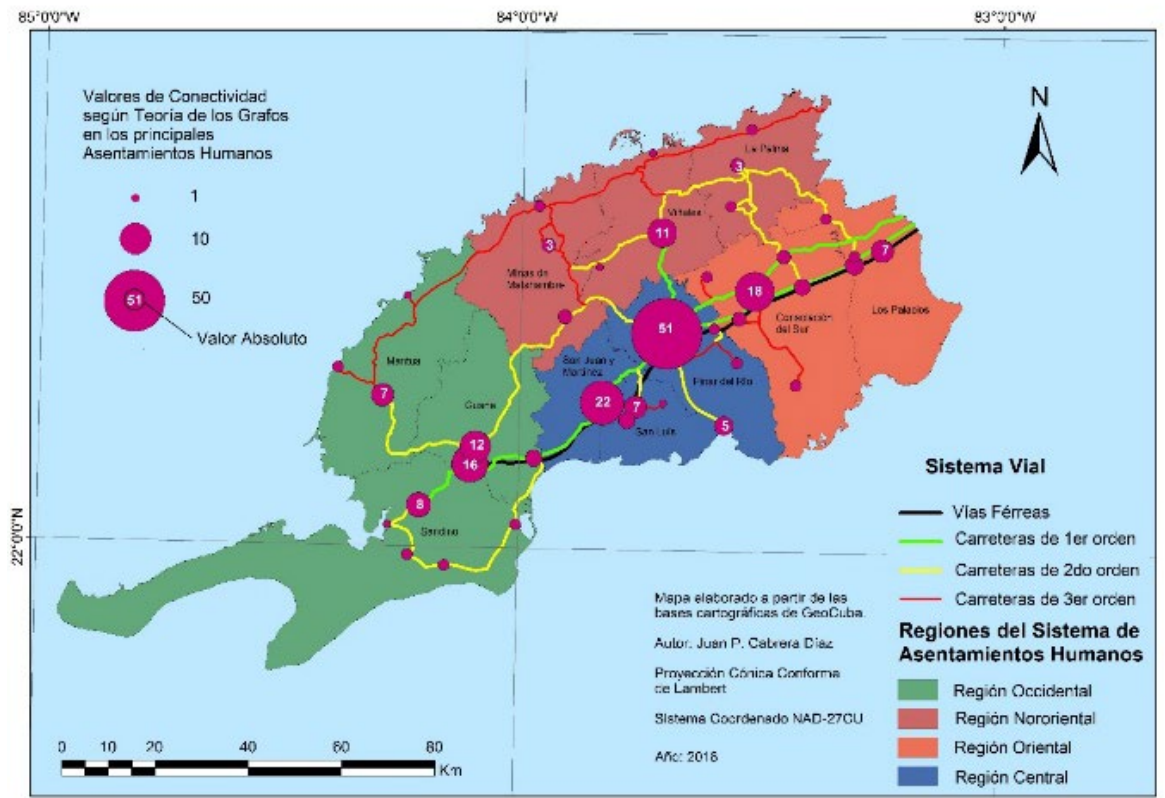

Figura 3: Mapa de Conectividad en Pinar del Río.

Fuente: Elaborado por el autor.

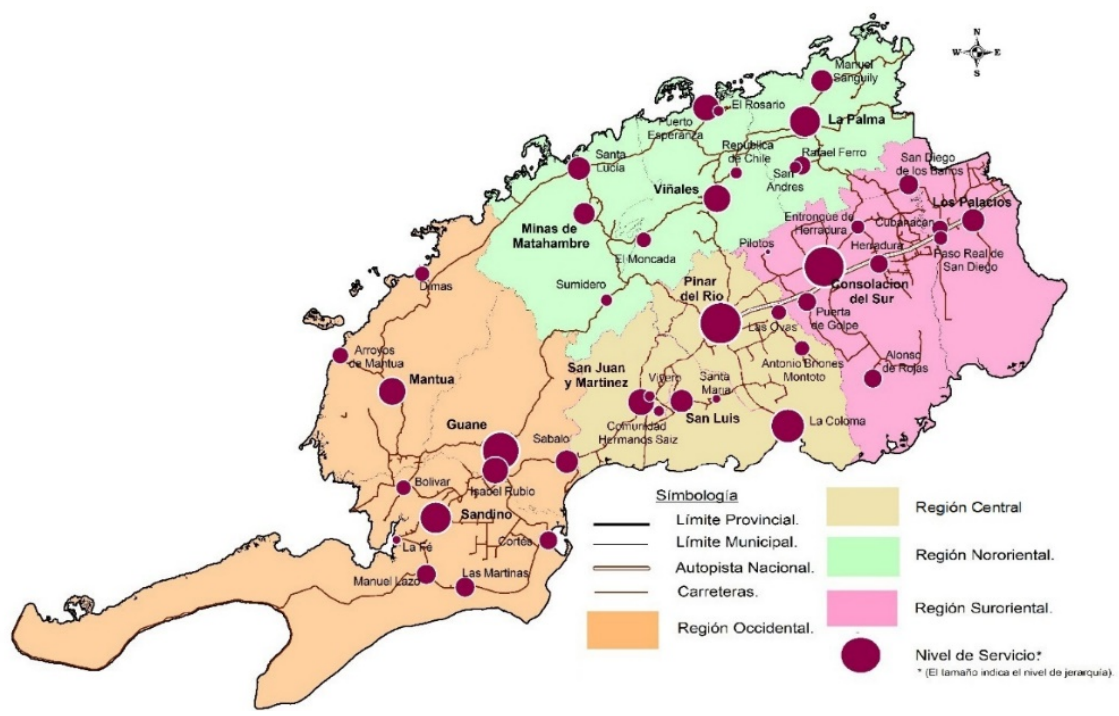

Figura 4. Jerarquía según nivel y cobertura de los servicios en Pinar del Río. Fuente: DPPF (2014). 


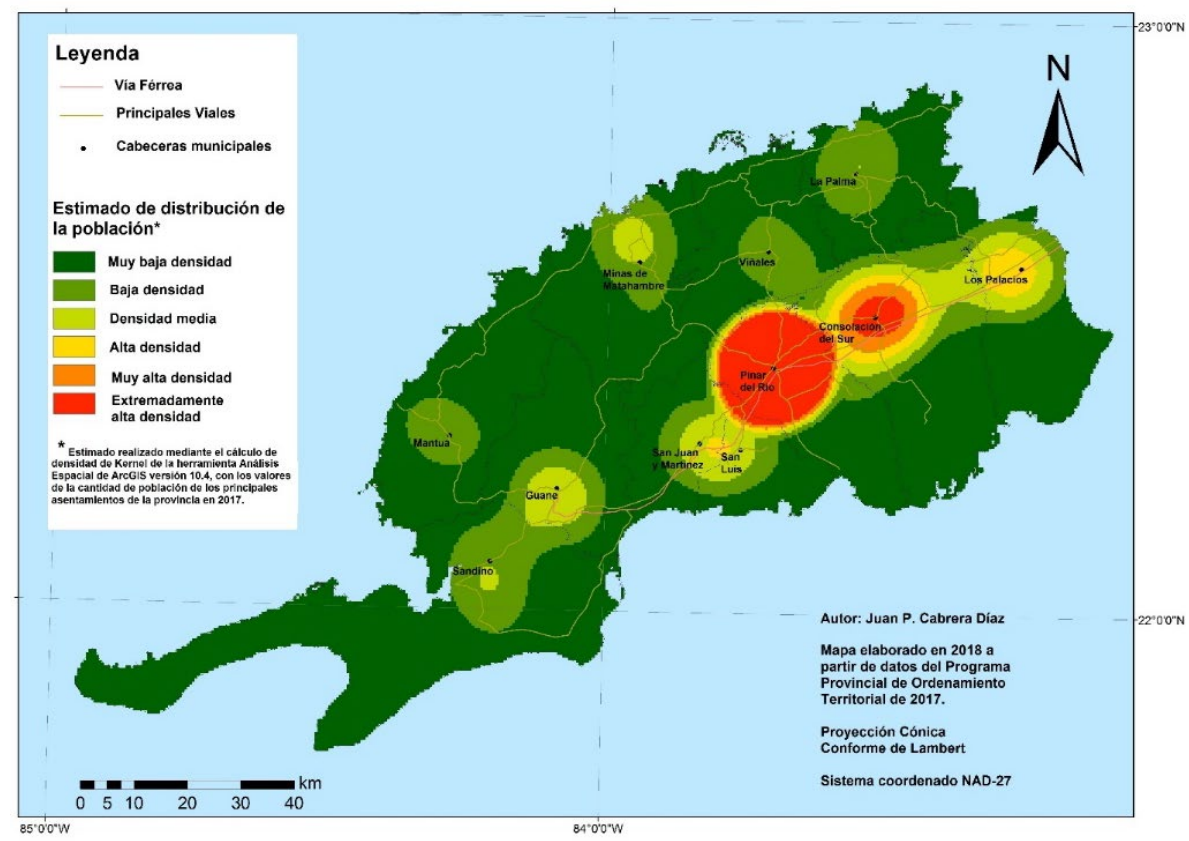

Figura 5. Mapa de distribución de la población en Pinar del Río.

Fuente: elaborado por el autor.

Al analizar los resultados obtenidos, resulta evidente la existencia de un centro polarizador de la población que concentra una muy alta densidad. Como es lógico, en el mismo se encuentran las dos principales ciudades, Pinar del Río y Consolación del Sur, en las que viven 168695 habitantes. Esta cifra absoluta resulta ser el $28.7 \%$ de la población total de la provincia, es decir, un tercio de los pinareños se concentran en un área que no alcanza los $400 \mathrm{~km}^{2}$. Este aspecto resulta más llamativo aún si se tiene en cuenta que el territorio más occidental del país tiene $8883.74 \mathrm{~km}^{2}$ de extensión.

En conclusión, teniendo en cuenta ambas ciudades, casi el $30 \%$ de la población de la provincia tiene su residencia en aproximadamente el 5\% del territorio de la misma. Si se analiza en función de los cuatro municipios que están en esa zona con alta densidad de población, háblese de San Juan y Martínez, San Luís, Pinar del Río y Consolación del Sur, entre ellos concentran al $59.6 \%$ de los habitantes de la provincia. Es decir, estos cuatro municipios presentan $3 / 5$ partes de la población. Claramente se puede hablar en este caso, de un sistema monocéntrico que se acentúa cada vez más, haciendo crecer la preponderancia de la ciudad capital sobre el resto de la provincia. 


\section{Consideraciones finales}

En el caso de Consolación, está condicionado su mal funcionamiento por diferentes aspectos que provocan el desaprovechamiento de sus potencialidades. Aunque las posibilidades de generar una mayor atracción están sustentadas en el alto nivel y cobertura existente en los centros de servicios de la ciudad, existen graves problemas de conectividad que atentan en ese sentido.

Lo analizado en los acápites anteriores indica la existencia de diferentes limitantes que condicionan que Consolación del Sur no funcione correctamente como centro intermunicipal de la región Oriental de Pinar del Río. Estos son:

- De los tres ejes viales de comunicación directa en el este de la provincia, solamente la Carretera Central atraviesa la ciudad Consolación del Sur, afectando sus relaciones funcionales con los territorios de su región.

- Como su principal vía de conexión con varios asentamientos de Los Palacios es mediante la Autopista Nacional, resulta mucho más cómodo para las personas que demandan los servicios, continuar hasta la capital provincial agregándole unos aproximados 30 minutos al viaje. De quedarse en Consolación del Sur, deben tomar un medio de transporte local de tipo privado (coche de caballos o bicitaxi) hasta el centro de la ciudad, el cual tarda casi el mismo tiempo del viaje hasta Pinar del Río. Esta misma situación se presenta si se utiliza como medio de transporte el ferrocarril que conecta esta parte del territorio provincial.

- Cercanía a la cabecera provincial, la cual genera una mayor atracción debido a su alto nivel de servicios.

- En la cabecera provincial, es posible encontrar ofertas mejores y más variadas de los muchos más servicios que en Consolación del Sur. Es algo normal debido a la diferencia en cuanto a la jerarquía en el sistema de asentamientos, sin embargo al Pinar del Río generar una mayor atracción y estar muy cerca geográficamente hablando, influye en el establecimiento de tendencias que establecen la no incorporación de Consolación del Sur a la satisfacción de necesidades de pobladores de otros asentamientos importantes en su región.

- Carencia de atractivos que hagan de la misma una ciudad dinámica.

- La mayoría de los atractivos que presenta la ciudad están en mal estado o en proceso de reparación desde hace varios años. Además, se puede incluir la falta de poder del gobierno local para propiciar la reparación de esos inmuebles o un desarrollo integral de la ciudad mediante un proceso inversionista que mejore las condiciones de la infraestructura y de los servicios.

- No existe un sistema de transporte efectivo que conecte a la ciudad Consolación del Sur con otros territorios de la región Oriental.

Esto se debe fundamentalmente a que el transporte del municipio está dominado en un alto por ciento por el sector cuentapropista y establece rutas en función de las demandas y no de las necesidades o de los planes de desarrollo 
estatales. En el caso del sistema de transportación estatal, no cuenta con los medios suficientes para generar un vínculo entre asentamientos de este nivel en el sistema, aspecto que dificulta la conectividad entre Consolación del Sur y la mayoría de los pueblos de la región Oriental, en especial los del municipio Los Palacios.

Sandino por su parte, cuenta con menor infraestructura que Consolación debido a que posee menor cantidad de habitantes. No obstante, hay que resaltar que en la región Occidental no existen centros poblados que semejen las condiciones de este último. También es esta región la que más problemas presenta por las condiciones que se han analizado en los acápites anteriores y la propuesta de que exista un centro que polarice los servicios pudiera mejorar la vinculación de la población con estos por la lejanía de la capital provincial.

Aun así, Sandino presenta varias limitantes que impiden que el mismo se desarrolle como centro regional. Entre ellas están:

- No existen un sistema de transporte que favorezca la conectividad en la ciudad, ni entre esta y los principales centros poblados de la región.

- Existe una mejor vinculación de los principales asentamientos de la región Occidental con la capital provincial que con su centro intermedio. Aunque la distancia es mucho mayor hasta Pinar del Río, hay una mejor conectividad debido a la existencia de rutas del sistema de transporte provincial. En un caso especial en ese sentido está Guane, pues presenta una conexión directa a través del ferrocarril y otras dos por la Vía Panamericana (la más usada) y por la Carretera Luís Lazo.

- Tramos de la Vía Panamericana en mal estado.

- Este aspecto dificulta la conectividad e impide el acercamiento de los pobladores de la región, en especial los que se mueven en medios propios y tienen la posibilidad de escoger sus opciones de movilidad. Además, es un tanto riesgoso para el transporte público y genera tardanzas indeseadas a los viajantes.

- Carencia de atractivos que hagan de la misma una ciudad dinámica.

- La mayoría de los atractivos que presenta la ciudad están en mal estado o en proceso de reparación desde hace varios años. Además, se puede incluir la falta de poder del gobierno local para propiciar la reparación de esos inmuebles o un desarrollo integral de la ciudad mediante un proceso inversionista que mejore las condiciones de la infraestructura y de los servicios.

- Presenta deficiencias en la red de acueducto, alcantarillado y comunicaciones y en la recogida de desechos.

- Estos problemas en las redes crean espacios deslucidos que fomentan la insalubridad y la vulnerabilidad ante determinadas circunstancias. Esto influye en la disfuncionalidad de los lugares públicos cercanos a ellos y 
proyectan una imagen poco llamativa de la ciudad que impacta en los niveles de atracción que la misma pueda generar.

\section{Conclusiones}

- La realización del análisis de Sandino y Consolación como centros intermedios del sistema de asentamientos en Pinar del Río, permitió determinar el carácter disfuncional de los mismos, debido a problemas de conectividad y a la ineficiencia de las infraestructuras de servicio.

- Los resultados del análisis determinan la existencia de una provincia monocéntrica, donde la capital provincial y los territorios cercanos a estas son los más favorecidos en cuanto a la satisfacción de necesidades y accesibilidad a los servicios. En este aspecto influye la disfuncionalidad de Sandino y Consolación como centros intermedios del SAH.

- La región Occidental es la que presenta menor desarrollo y la menos funcional es la región Nororiental, debido a que la población de la misma está más vinculada a la capital provincial que a los centros de servicios que existen en la misma, especialmente en el caso de los mineros y los palmeros.

- La inexistencia de un sistema de transporte que conecte los principales centros poblacionales de las regiones Occidental y Oriental, con sus centros intermedios, dificulta la movilidad de la población y genera movimientos pendulares más fuerte hasta la ciudad de Pinar del Río, en la cual existe una red de rutas de transportación desde y hacia varios pueblos de la provincia.

- Se deben generar procesos de desarrollo en ambos centros intermedios que realcen la visibilidad de los mismos en sus regiones, haciéndolos más atractivos y eficientes mediante inversiones que permitan crear infraestructuras de servicios y mejorar las ya existentes, promoviendo un vínculo a través de sistemas de transporte eficaces.

\section{Bibliografía}

Bermúdez Mulet, E., Procedimiento para el análisis de los centros intermedios, Instituto de Planificación Física, no publicado, 2015.

—_ Ciudades de Interés Nacional, La Habana, Cuba, 2000.

Bermúdez Mulet, E. \& Boquet García, A., "Caracterización del sistema de asentamientos de Cuba”, Revista de Ordenamiento Territorial y Urbanismo, Cuba, 2013.

Beuf Hill, J., "Los asentamientos humanos y su jerarquía. Un acercamiento a la pampa argentina”, Ediciones Río La Plata, Argentina, 2015.

González Rego, R., "Geografía Urbana”, Editorial Félix Varela, La Habana, Cuba, 2016.

Haggett, P., "Análisis Locacional en la Geografía Humana”, Colección Ciencia Urbanística 17, Editorial Gustavo Gili, Barcelona, España, 1976.

Higueras Arnal, A., "Teoría y Método de la Geografía. Introducción al análisis geográfico regional”, Colección textos docentes, Prensas Universitarias de Zaragoza, España, 2003.

IPF, “Plan Provincial de Ordenamiento Territorial”, Pinar del Río, Cuba, 2014. 
__., "Esquema Nacional de Ordenamiento Territorial”, La Habana, Cuba, 2015. , "Plan Provincial de Ordenamiento Territorial”, Pinar del Río, Cuba, 2017.

__, "Programa General de Ordenamiento Territorial Urbano. Consolación del Sur”, Pinar del Río, Cuba, 2014.

_- "Programa General de Ordenamiento Territorial Urbano. Sandino", Pinar del Río, Cuba, 2014.

"Jerarquía y áreas de influencia de localidades del estado de México", Revisado en: http://observatoriogeograficoamericalatina.org.mx/egal4/Geografiasocioeconom ica/Geografiaurbana/12.pdf. Recuperado el 25 de junio del 2017.

"La Teoría de los Lugares Centrales de Walter Christaller", Revisado en: http://eumed.net/economics_teories/christaller.pdf. Recuperado el 25 de junio de 2017.

Llop, H., "Territorial Sistems and own develop”. Ottawa University, Canadá, 2013.

Lobato Correa, R. \& Rosendahl, Z., "Introdução a Geografia Cultural”, Orgs. Bertand Brasil, Rio de Janeiro, Brasil, 2002.

Mateo Rodríguez, J. \& Celeiro Chaple, M., "Cambios Espaciales en América Latina y su incidencia en los sistemas ambientales", A conferência da terra, Fórum Internacional do Meio Ambiente, João Pessoa, Estado de Paraíba, Brasil, 2009.

Michelini, J. J., "Fundamentos conceptuales y metodológicos del ordenamiento territorial”, Bogotá, Colombia, 2009. 